lanburton_10@hotmail.co.uk

\title{
A philosophy of transformational leadership may be the optimal approach for enhanced outcomes in healthcare and physiotherapy settings: A narrative review
}

Ian Burton MSc, CSCS

Specialist Musculoskeletal Physiotherapist, NHS Grampian

Please cite as: Burton, I. (2021). A philosophy of transformational leadership may be the optimal approach for enhanced outcomes in healthcare and physiotherapy settings: A narrative review

SportRxiv doi: 


\section{Introduction}

Modern working in healthcare settings such as the National Health Service (NHS) is heavily reliant on the adoption of evidence-based medicine and practice, which is seen as essential to effective working, high productivity and increased quality of care. However, leadership of healthcare staff can be considered equally as important for improving the integration and quality of care provided in healthcare settings such as the NHS. Quality of care is an integral component of achieving high productivity within healthcare organisations and is defined as the degree to which the probability of achieving the expected health outcomes is increased and in line with updated professional knowledge and skills within health services (Institute of Medicine 2001). The measurement of health outcomes is considered a core component of measuring quality care. The Institute of Medicine has described six characteristics of highquality care that must be: (1) safe, (2) effective, (3) reliable, (4) patient-centred, (5) efficient, and (6) equitable. Quality measures include structure, process, outcome, and patient satisfaction (Institute of Medicine 2001). Measures on patient outcomes and satisfaction constitute shorter patient length of stay, hospital mortality level, healthcare-associated infections, failure to rescue ratio, restraint use, medication errors, inadequate pain management, pressure ulcers rate, patient fall rate, falls with injury, medical errors, and urinary tract infections (Lang 2007).

Leadership can be considered as the relationship between those who lead and those who take the choice to follow, and directly refers to the behaviour of directing and coordinating the activities of a team or group of people towards a common goal (Kousez and Posner 2002). There are numerous publications recognising an effective leadership style as a key element for implementing quality healthcare and leading an organisation to effective and successful outcomes (Al-Sawai 2013). Significant positive associations between effective styles of leadership and high levels of patient satisfaction and reduction of adverse effects have been reported in the literature. Several studies have stressed the importance of leadership style for quality of healthcare provision in various settings (Sfantou et al. 2017). The aim of this review is to give an overview of the most common leadership styles adopted in healthcare settings, with a particular focus on the strengths and weaknesses of the two most common approaches: transactional and transformational leadership. The review will conclude with an overview of recent evidence which builds an argument for transformational leadership as the most effective style for improved outcomes for patients and staff 
satisfaction and performance within healthcare settings, with a particular focus on physiotherapy settings, as the author currently works as a physiotherapist within the NHS.

\section{Leadership models in healthcare}

In order to influence patient care, improve healthcare outcomes, enhance performance of clinical staff and optimise future directions of healthcare organizations, healthcare workers need to become effective leaders in all healthcare settings. There is a significant body of evidence which indicates that effective leadership approaches in healthcare improves clinical outcomes for patients and enhances staff engagement through improved well-being and reduced staff burnout (Cochran et al. 2014; Ham 2003; Majmudar et al. 2010; Shanafelt and Noseworthy 2017; Shanefelt et al. 2015). Hargett et al. (2017) defines healthcare leadership as "the ability to effectively and ethically influence others for the benefit of individual patients and populations". The most common types of leadership identified in healthcare settings include transactional, autocratic, laissez-faire, task-oriented, relationship-oriented leadership, and the increasingly prevalent transformational leadership (Sfantou et al. 2017). In transactional leadership the leader acts as a manager of change, making exchanges with employees that lead to an improvement in production (Burns, 1978). The transactional leadership style has been the traditional approach adopted in hierarchical healthcare settings, however recently it has become less favourable compared to a transformational leadership approach, due to several key differences (Table 1). An autocratic leadership style is considered ideal in an emergency medical situation as the leader makes all decisions without considering the opinion of staff, with mistakes not tolerated and blame put on individuals. In contrary, the laissez-faire leadership style involves a leader who does not make decisions, staff acts without direction or supervision with a hands-off approach resulting in rare changes (Frandsen, 2014). Task-oriented leadership style involves planning of work activities, clarification of roles within a team or a group of people, objectives set as well as the continuing monitoring and performance of processes. In contrast, a Relationshiporiented leadership style incorporates support, development and recognition (Yukl, 2010). 
Table 1: Kotter (1990) comparison of transactional and transformational leadership

\begin{tabular}{|l|l|l|}
\hline Creating agenda & Transactional leadership & Transformational leadership \\
\hline $\begin{array}{l}\text { Developing Human } \\
\text { Resources }\end{array}$ & $\begin{array}{l}\text { Planning and budgeting: } \\
\text { developing a plan - a } \\
\text { detailed map of how to } \\
\text { achieve the results }\end{array}$ & $\begin{array}{l}\text { Establishing direction: } \\
\text { developing direction - a } \\
\text { vision which describes a } \\
\text { future state along with a } \\
\text { strategy }\end{array}$ \\
\hline Execution & $\begin{array}{l}\text { Organising and staffing: } \\
\text { which individual best fits } \\
\text { each job and what part of } \\
\text { the plan fits each individual }\end{array}$ & $\begin{array}{l}\text { Aligning people: a major } \\
\text { communication challenge } \\
\text { getting people to understand } \\
\text { and believe the vision }\end{array}$ \\
\hline $\begin{array}{l}\text { Controlling and problem- } \\
\text { solving: monitoring results } \\
\text { - identifying deviations from } \\
\text { the plan and solving the } \\
\text { problems }\end{array}$ & $\begin{array}{l}\text { Motivating and inspiring: } \\
\text { satisfying basic human needs } \\
\text { for achievement, belonging, } \\
\text { recognition, self-esteem, a } \\
\text { sense of control }\end{array}$ \\
\hline Outcomes & $\begin{array}{l}\text { Produces degree of } \\
\text { predictability and order }\end{array}$ & $\begin{array}{l}\text { Produces changes - often to } \\
\text { a dramatic degree }\end{array}$ \\
\hline
\end{tabular}

Leaders must also possess certain characters (Figure 1) in order to be suited for leadership roles and particular leadership styles (Crossan et al. 2016). Leader character and particularly the role played by the dimension of judgement may renew situational theories of leadership. This set of theories focuses on the characteristics of the situation (e.g., task characteristics or features of interpersonal relationships) that should determine the appropriate leadership style. Hence effective leaders must be situationally aware, insightful, adaptable and demonstrate good judgement, which contributes to the success of a team or organization (Crossan et al. 2016). For example, Bass (1995) clarified that transformational leadership is not a pure style, as transformational leaders can display transactional behaviours if warranted. The two approaches are not mutually exclusive, with individual leaders potentially possessing varying elements of both transformational and transactional leadership approaches, with a combination of elements from both approaches potentially enhancing leadership effectiveness (Aarons 2006). Thus, judgement is central in orchestrating and activating the character dimensions as required by the situation. The dimensions of 
character may therefore explain why some leaders have versatility to lead across different contexts.

Figure 1: Leader characters (Crossan et al. 2016)

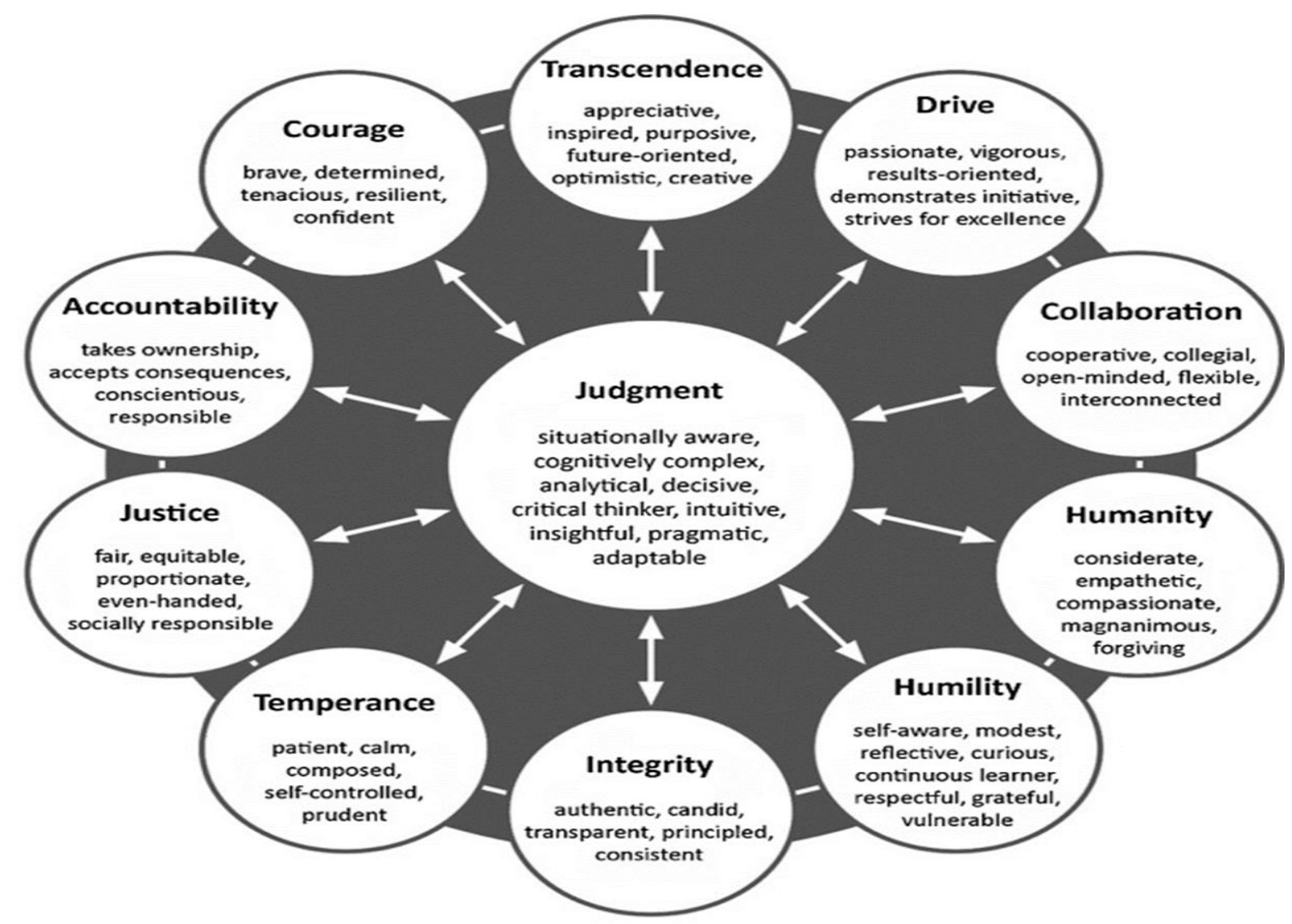

Source: Reproduced from Crossan et al. (2016)

A recent study aimed to investigate what competencies are required for effective leadership in healthcare, based on the development of a conceptual model specific to the healthcare setting. The resulting model (Figure 2) features the central core principle of Patient Centeredness which is surrounded by an overlapping five core competencies. Emotional Intelligence is considered the core competency that holds the other competencies together, and therefore it is positioned as the "keystone" in the model; if Emotional Intelligence is removed, the authors believe the model will crumble (Bradberry and Greaves 2009). Integrity and Selfless Service are intentionally positioned at the base of the model, although they may be difficult to teach, they are extremely important to effective healthcare leadership and must 
be recognized and emphasized as essential "foundational" core competencies (Hargett et al. 2017). Critical Thinking and Teamwork are positioned as the "framework core competencies", holding the model together and overlapping with the other three competencies (Hargett et al. 2017). There is now a growing body of evidence highlighting the specific leadership characters and competencies required in healthcare, however the specific overall leadership approach which is most applicable for their successful implementation is unclear. The remainder of this review will present an argument for transformational leadership as being the optimal leadership approach for incorporating these essential competencies in healthcare as compared to the traditional transactional leadership approach.

Figure 2: Competencies for effective leadership in healthcare (Hargett et al. 2017)

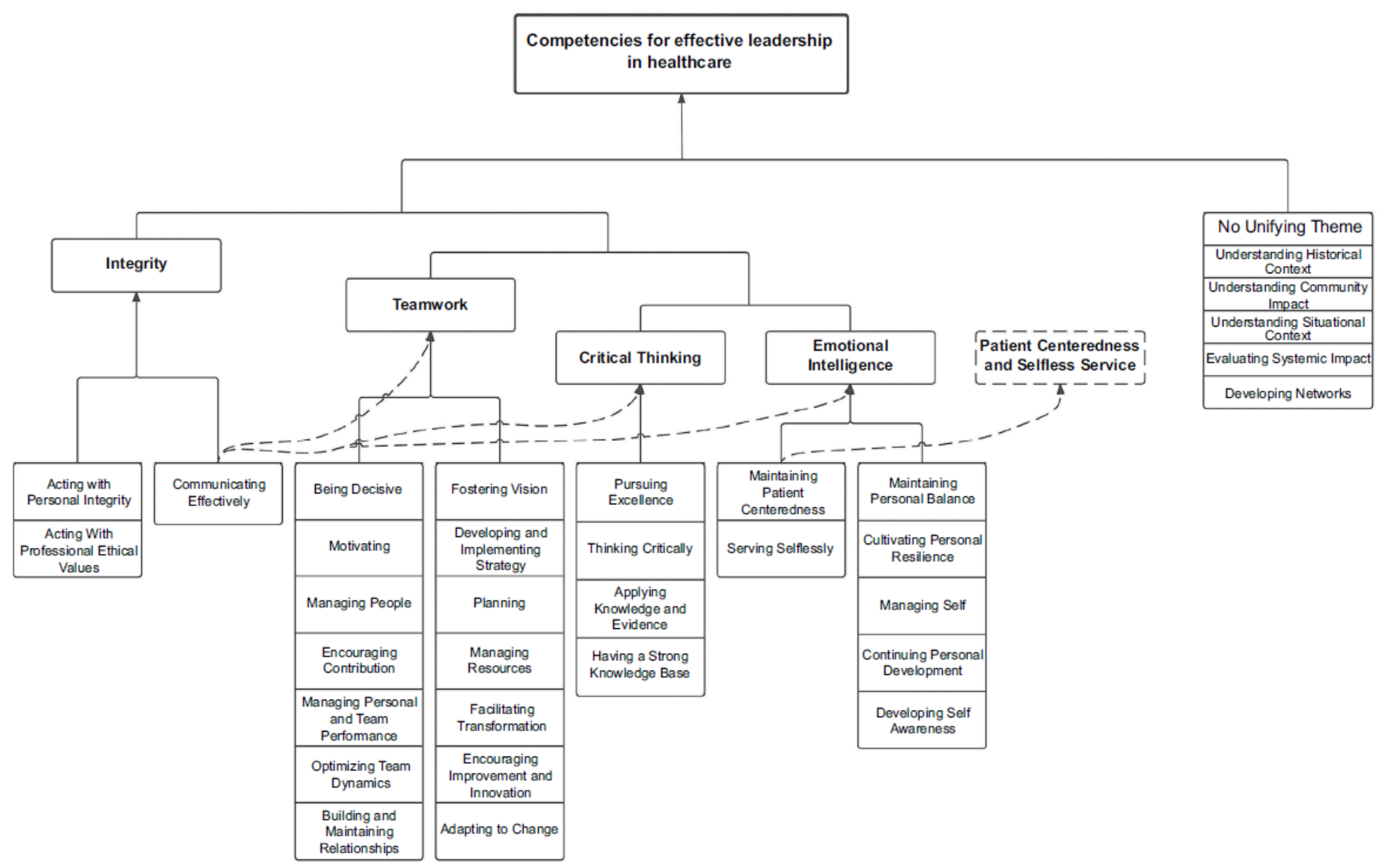




\section{Comparison of Transactional and Transformational leadership}

Transformational leadership is an approach to leadership which aims to inspire and stimulate followers to not only achieve extraordinary outcomes above and beyond the status quo, but to also develop their own capacity for leadership responsibility (Bass and Riggio 2006). Leaders who adopt this approach are often considered to be enthusiastic, passionate, energetic, and emotionally intelligent, which are considered prerequisite characteristics of a transformational leader (Horwitz et al. 2008). Transformational leaders are not only actively involved in the leadership process but are genuinely concerned and focused on helping team members succeed (Alkassabi et al. 2018). In contrast, transactional leadership is more like a typical managerial leadership approach, which focuses on the roles of the organization, supervision and staff performance. Transactional leaders are driven by a focus on specific task completion, based on a system of rewards and punishments to extrinsically motivate staff (Horwitz et al. 2008). A key advantage of the transactional leadership approach is that it is based on clearly defined roles in which individuals know what they must do and what rewards they will receive in exchange for task completion (Alkassabi et al. 2018). The focus on extrinsic reward can allow leaders to supervise attentively and motivate staff to meet expected levels of performance to achieve the desired rewards (Gill 2011).

Transformational leadership may be compared to charismatic or visionary leadership (Howell 1997), as leaders adopting this approach are often considered capable of inspiring and motivating others in ways beyond the typical goals of exchanges and rewards (House et al. 1991). Settings which consist of close supervisory relationships are considered particularly suitable to a transformational leadership approach, compared to those in which more distant relationships exist with closer supervision by mentors often more typical in hierarchical healthcare settings (Howell and Hall-Merenda 1999). This close relationship may be typical of a supervisor-supervisee relationship in healthcare settings which is represented by the term "first-level leaders", who are thought to be important because of their functional proximity to supervisees (Priestland and Hanig 2005). Transformational leadership has been found to increase intrinsic motivation in healthcare staff (Gardiner and Avolio 1998) through the expression of the value and importance of the leader's goals (Bass and Avolio 2003). In contrast, transactional leadership is based more on "exchanges" between the leader and supervisees, in which staff are primarily rewarded for meeting specific goals or performance criteria, complimented by positive reinforcement or tangible rewards by leadership (Waldman et al. 1990, Jung 2001). This emphasis on meeting specific targets or objectives within a 
transactional leadership approach, means that it is often considered more practical in nature, in comparison to transformational leadership (Avolio and Bass 1988). An effective transactional leader can recognize and reward subordinates' accomplishments and take necessary action in a timely fashion, however this may come at the expense of innovative thinking due to a reliance on monitoring performance based on stringent pre-determined criteria. However, less competent transactional leaders may be less likely to anticipate or identify problems and take the necessary actions in a timely manner (Jung 2001).

Although transactional leadership is an appropriate approach in many settings as it can support adherence to specific standards and targets, there is a distinct lack of openness to innovation, which can be considered a significant weakness in today's ultra-competitive marketplace (Jung 2001). However, a transformational leadership style does not share this weakness as it creates a vision based on intrinsic motivation, which inspires staff to strive beyond the required expectations or pre-determined standards. In comparison, transactional leadership heavily focuses on rewards and achievements based on extrinsic motivation for the completion and performance of job tasks (Amabile 1998). Transformational leadership is therefore more likely to influence attitudes by inspiring acceptance of innovation through the development of characteristics such as enthusiasm, trust, and openness. However, a transactional leadership approach would be limited to the acceptance of innovation through implementing reinforcement and reward. In today's workplace environment, leadership is important to consider in relation to the acceptance of innovation, due to its necessity in competition and survival, even in public sector organisations such as the NHS, due to reduced funding, budget constraints and closing of financially unsustainable services. The leadership approach taken and its openness to innovation can have direct applicability to work attitudes, perceptions, behaviour, service quality, and client outcomes (Aarons 2006). As leadership style is associated with organizational, team and staff performance, it is likely to have a direct influence on healthcare manager's and service leaders' attitudes toward adoption of evidence-based practices, which are now considered essential in healthcare practice (Aarons 2006).

\section{Transformational leadership in healthcare}

The transformational leadership style has become widely implemented in healthcare and is characterized by creating relationships and motivation among staff members. 
Transformational leaders typically can inspire confidence and staff respect, and they communicate loyalty through a shared vision, resulting in increased productivity, strengthened employee morale, and job satisfaction (Sfantou 2017). Transformational leadership is strongly related to the implementation of effective management that establishes a culture of patient safety (Page 2004). Transformational leadership stresses the importance of empowering others, which is related to patient outcomes by promoting greater staff expertise through increased staff stability and preventing reduced turnout (Houser 2003). Effective leadership has an indirect impact on reducing mortality rates, by inspiring, retaining and supporting experienced staff. For these reasons, it appears that the transformational leadership style is not only the most effective style in healthcare in terms of outcomes but also the most appropriate for the modern healthcare environment. Bass and Avolio (1990) consider transformational leadership to have four key components; individual influence, inspirational motivation, intellectual stimulation and individualised consideration (Figure 3). These components when combined with the traditional transactional leadership components of contingent reward and management by exception can have an additive effect on expected outcomes, by improving performance beyond expectations (Bass and Avolio 1990).

Figure 3: Components of transformational leadership (Bass and Avolio 1990)

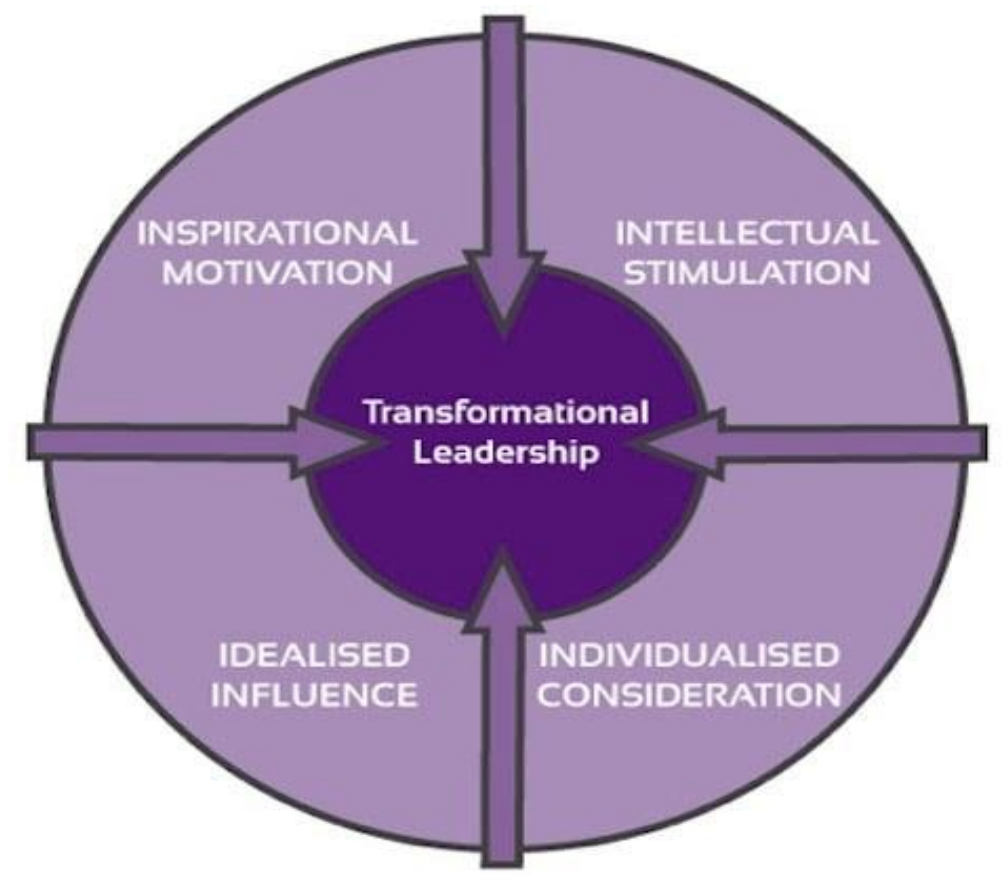

The style adopted in transformational leadership may be characterized by creating positive relationships and increasing motivation among staff by inspiring confidence, respect and 
loyalty through communicating a shared vision for change and organisational direction (Frandsen 2014; Bass \& Avolio 2006). Transformational leadership styles that conceptualize management as a collaborative approach and are employee-oriented are consistently found to be most effective in healthcare settings. A recent systematic review, which included 18 studies found transformational leadership was more effective than other styles, leading to better healthcare outcomes and well-coordinated and integrated provision of quality care (Sfantou et al. 2017). Examination of the literature identifies a plethora of positive outcomes from a transformational leadership stye including increased healthcare organisational culture and staff empowerment, higher levels of job satisfaction and commitment, higher productivity, staff retention and morale, patient safety, quality care and positive health outcomes (Casida et al. 2008; Armellino et al. 2010; Chan et al. 2015; Moneke et al. 2013; Khan et al. 2018; Alkassabi et al. 2018; Alloubani et al. 2019). Further to this, transformational leadership has been found to enhance psychological empowerment, knowledge sharing, and intrinsic motivation which can foster innovative and proactive work behaviour in healthcare workers (Masood and Afsar 2017; Steinmann et al. 2018).

A recent cross-sectional survey study conducted with physiotherapists in Saudi Arabia (Alkassabi et al. 2018) found that physiotherapists had ambivalent responses in ratings of job satisfaction regardless of whether they worked in the public or private sector. Job satisfaction was highly correlated to issues involving pay, promotion, benefits, contingent rewards and communication, with participants being undecided regarding satisfaction with these factors. However, job satisfaction was higher in relation to their immediate supervisor's leadership style, co-workers and the nature of their work. The findings contrast that of a Turkish study in which 54\% of 198 physiotherapists were dissatisfied with their jobs, especially in relation to salary and advancement opportunities, which they linked to leadership quality (Eker et al. 2004). Physiotherapists in this study reported a significant positive relationship between the perception of leadership quality received and their levels of job satisfaction and considered leadership quality the best predictor of job satisfaction. Leadership style has also been shown to have a significant effect of job satisfaction in the nursing profession. Negussie and Demissie (2013) found that Nurses prefer transformational leadership style over transactional leadership style and had moderate-level intrinsic but low level of extrinsic job satisfaction in a survey of 175 nurses. Furthermore, from transactional leadership, only contingent reward was found to be statically significant and correlated with extrinsic and intrinsic job satisfaction, while all five dimension of transformational leadership style were statistically significant and correlated with both intrinsic and extrinsic job satisfaction. 
The findings of Alkassabi et al. (2018) are like other studies conducted with physiotherapists, which indicate job satisfaction is linked to salary, benefits, recognition, opportunities, communication and leadership styles (Aleisa et al. 2015, Decker et al. 2009; Judge and Bono 2011; Johanson 2007; Rozier et al. 1998; Arkwright et al. 2018; Bacopanos and Edgar 2016; Chao et al. 2015; Haybatollahi et al. 2014; Vander Elst et al. 2016). These factors may also be related to job autonomy, which is shown to be highly correlated with job satisfaction and can be determined by workplace leadership styles (Vaananen et al. 2005).

Physiotherapist's perceptions of aspects of their jobs can be associated with intrinsic factors such as self-determination and perceived competence (Decker et al. 2009). Therefore, methods for improving job satisfaction, performance and well-being should consider not only extrinsic factors but also intrinsic factors such as perceived self-esteem, self-efficacy, locus of control and emotional stability, all of which can be increased by adopting a transformational leadership approach (Judge and Bono 2009). The study by Alkassabi et al. (2018) found that leadership style is significantly associated with job satisfaction in physiotherapists, with a strong positive correlation between the two variables, which has also been found in other studies (Mosadeghrad et al. 2011; Chen et al. 2012). The physiotherapists were satisfied with their supervisors and perceived their leadership style to be more of a transformational approach rather than a transactional or passive/avoidant style. Although further research from larger cohorts in different countries around the world is required to increase the generalisability of these findings, there is a growing body of evidence suggesting managers can improve employee job satisfaction, commitment and productivity in healthcare through adopting transformational leadership approaches (Mosadeghrad et al. 2011; Kuoppala et al. 2008; Gui et al. 2009). Wylie and Gallagher (2009) suggest that physiotherapists' approach to inspiring and empowering others makes them "naturally advantaged" to practice transformational leadership, despite their being a significant dearth of research on leadership in physiotherapy (Desveaux et al. 2016; McGowan 2018).

Although most of the research on transformational leadership in healthcare has been conducted outside the United Kingdom (UK), there have been some studies which provide examples of the implementation of this approach in a local healthcare context. A transformational leadership style has been found to enhance performance in 'extended or advanced roles' which increases hospital efficiency, due to increased self-efficacy and engagement levels (Salanova et al. 2011; McCaffrey et al. 2017). This is a significant finding, considering that NHS Scotland is currently implementing widespread adoption of such roles, particularly in the physiotherapy profession, including the increase in first contact 
physiotherapist practitioner roles in general practitioner (GP) practices. A study conducted within NHS Scotland found that a transformational leadership style within a nursing department led to sharing of leadership roles across teams, with staff being more engaged, leading to increased quality effective care and meeting policy change targets, ensuring quality sustainable NHS services (Tomlinson 2012).

\section{Conclusion}

This review highlights the importance of leadership within modern healthcare settings and provided an overview of common healthcare leadership approaches, including transactional and transformational leadership. Research has identified leader characters and specific competencies which are required by leaders in healthcare in order to achieve optimal outcomes. Although both transactional and transformational leadership approaches have their strengths and weaknesses, the evidence presented suggests a transformational leadership approach better suits the specific competencies required within a healthcare leadership context. Interventional, survey and qualitative studies have found a plethora of benefits associated with a transformational leadership approach in healthcare, including better patient outcomes and higher staff satisfaction and motivation compared to other approaches to leadership. Studies conducted with nursing and physiotherapy staff have consistently shown better outcomes with a transformational leadership approach, which is significant as they represent the second and third largest healthcare professions by numbers employed, respectively. Transformational leadership therefore appears to be the most evidence-based approach available within healthcare, at a time when evidence-based practice is considered mandatory within the NHS. Effective leadership within the NHS has never been more critical than at present, due to reduced government spending in healthcare and the increased pressures on the system during the Covid-19 pandemic. Although a transactional leadership approach in isolation has several weaknesses in modern healthcare and may be considered outdated, some of its components still possess merit and may have an additive effect if implemented alongside an overall transformational leadership approach in order to achieve optimal outcomes for patients and staff in healthcare settings. 


\section{References}

AARONS, G.A., 2006. Transformational and transactional leadership: association with attitudes toward evidence-based practice. Psychiatric services (Washington, D.C.), 57(8), pp. $1162-1169$

ALEISA, E. et al., 2015. Predictors of global job satisfaction among Saudi physiotherapists: a descriptive study. Annals of Saudi medicine, 35(1), pp. 46-50

ALKASSABI, O.Y. et al., 2018. Job satisfaction among physiotherapists in Saudi Arabia: does the leadership style matter? BMC health services research, 18(1), pp. 422-018-3184-9

ALLOUBANI, A. et al., 2019. Leadership styles' influence on the quality of nursing care. International journal of health care quality assurance, 32(6), pp. 1022-1033

AMABILE, T.M., 1998. How to kill creativity. Harvard business review, 76(5), pp. 76-87, 186

ARMELLINO, D., QUINN GRIFFIN, M.T. and FITZPATRICK, J.J., 2010. Structural empowerment and patient safety culture among registered nurses working in adult critical care units. Journal of nursing management, 18(7), pp. 796-803

BACOPANOS, E. and EDGAR, S., 2016. Identifying the factors that affect the job satisfaction of early career Notre Dame graduate physiotherapists. Australian Health Review : A Publication of the Australian Hospital Association, 40(5), pp. 538-543

BASS, B.M. et al., 2003. Predicting unit performance by assessing transformational and transactional leadership. The Journal of applied psychology, 88(2), pp. 207-218

CASIDA, J., 2008. Linking nursing unit's culture to organizational effectiveness: a measurement tool. Nursing economic\$, 26(2), pp. 106-110

CHAN, Z. et al., 2015. What Makes a Leader: Identifying the Strengths of Canadian Physical Therapists. Physiotherapy Canada.Physiotherapie Canada, 67(4), pp. 341-348

CHAO, M.C. et al., 2015. Workplace stress, job satisfaction, job performance, and turnover intention of health care workers in rural Taiwan. Asia-Pacific journal of public health, 27(2), pp. NP1827-36 
CHEN, A.H., JAAFAR, S.N. and NOOR, A.R., 2012. Comparison of job satisfaction among eight health care professions in private (non-government) settings. The Malaysian journal of medical sciences : MJMS, 19(2), pp. 19-26

DECKER, F.H., HARRIS-KOJETIN, L.D. and BERCOVITZ, A., 2009. Intrinsic job satisfaction, overall satisfaction, and intention to leave the job among nursing assistants in nursing homes. The Gerontologist, 49(5), pp. 596-610

DESVEAUX, L., CHAN, Z. and BROOKS, D., 2016. Leadership in Physical Therapy: Characteristics of Academics and Managers: A Brief Report. Physiotherapy Canada.Physiotherapie Canada, 68(1), pp. 54-58

DESVEAUX, L. and VERRIER, M.C., 2014. Physical Therapists' Perceptions of Leadership across the Health Care Continuum: A Brief Report. Physiotherapy Canada.Physiotherapie Canada, 66(2), pp. 119-123

EKER, L. et al., 2004. Predictors of job satisfaction among physiotherapists in Turkey. Journal of occupational health, 46(6), pp. 500-505

GUI, L., BARRIBALL, K.L. and WHILE, A.E., 2009. Job satisfaction of nurse teachers: a literature review. Part II: Effects and related factors. Nurse education today, 29(5), pp. 477487

HAM, C., 2003. Improving the performance of health services: the role of clinical leadership. Lancet (London, England), 361(9373), pp. 1978-1980

HARGETT, C.W. et al., 2017. Developing a model for effective leadership in healthcare: a concept mapping approach. Journal of healthcare leadership, 9, pp. 69-78

HAYBATOLLAHI, M. and GYEKYE, S.A., 2014. The moderating effects of locus of control and job level on the relationship between workload and coping behaviour among Finnish nurses. Journal of nursing management, 22(6), pp. 811-821

HORWITZ, I.B. et al., 2008. Transformational, transactional, and passive-avoidant leadership characteristics of a surgical resident cohort: analysis using the multifactor leadership questionnaire and implications for improving surgical education curriculums. The Journal of surgical research, 148(1), pp. 49-59 
JOHANSON, M.A., 2007. Sex differences in career expectations of physical therapist students. Physical Therapy, 87(9), pp. 1199-1211

JUDGE, T.A. and BONO, J.E., 2001. Relationship of core self-evaluations traits--selfesteem, generalized self-efficacy, locus of control, and emotional stability--with job satisfaction and job performance: a meta-analysis. The Journal of applied psychology, 86(1), pp. 80-92

KHAN, B.P., QUINN GRIFFIN, M.T. and FITZPATRICK, J.J., 2018. Staff Nurses' Perceptions of Their Nurse Managers' Transformational Leadership Behaviors and Their Own Structural Empowerment. The Journal of nursing administration, 48(12), pp. 609-614

KOTTER, J.P., 1988. The leadership factor. New York; London: Free Press; Collier Macmillan.

MAJMUDAR, A. et al., 2010. High-performance teams and the physician leader: an overview. Journal of surgical education, 67(4), pp. 205-209

MASOOD, M. and AFSAR, B., 2017. Transformational leadership and innovative work behavior among nursing staff. Nursing inquiry, 24(4), pp. 10.1111/nin.12188. Epub 2017 Feb 2

MCCAFFREY, R. and REINOSO, H., 2017. Transformational Leadership: A Model for Advanced Practice Holistic Nurses. Journal of holistic nursing : official journal of the American Holistic Nurses' Association, 35(4), pp. 397-404

MCGOWAN E PHD, ,P.T., ELLIOTT N PHD, ,R.N. and STOKES E PHD, ,P.T., 2018. Leadership capabilities of physiotherapy leaders in Ireland: Part 1 physiotherapy managers. Physiotherapy theory and practice, , pp. 1-17

MCGOWAN, E., ELLIOTT, N. and STOKES, E., 2018. Leadership capabilities of physiotherapy leaders in Ireland: Part 2. Clinical specialists and advanced physiotherapy practitioners. Physiotherapy theory and practice, , pp. 1-17

MONEKE, N. and UMEH, O.J., 2013. How leadership behaviors impact critical care nurse job satisfaction. Nursing management, 44(1), pp. 53-55 
MOSADEGHRAD, A.M., FERLIE, E. and ROSENBERG, D., 2008. A study of the relationship between job satisfaction, organizational commitment and turnover intention among hospital employees. Health services management research, 21(4), pp. 211-227

MOSADEGHRAD, A.M., FERLIE, E. and ROSENBERG, D., 2011. A study of relationship between job stress, quality of working life and turnover intention among hospital employees. Health services management research, 24(4), pp. 170-181

NEGUSSIE, N. and DEMISSIE, A., 2013. Relationship between leadership styles of nurse managers and nurses' job satisfaction in Jimma University Specialized Hospital. Ethiopian journal of health sciences, 23(1), pp. 49-58

PRIESTLAND, A. and HANIG, R., 2005. Developing first-level leaders. Harvard business review, 83(6), pp. 112-20, 150

ROZIER, C.K. et al., 1998. Gender and physical therapy career success factors. Physical Therapy, 78(7), pp. 690-704

SALANOVA, M. et al., 2011. Linking transformational leadership to nurses' extra-role performance: the mediating role of self-efficacy and work engagement. Journal of advanced nursing, 67(10), pp. 2256-2266

SFANTOU, D.F. et al., 2017. Importance of Leadership Style towards Quality of Care Measures in Healthcare Settings: A Systematic Review. Healthcare (Basel, Switzerland), 5(4), pp. 10.3390/healthcare5040073

SHANAFELT, T.D. et al., 2015. Impact of organizational leadership on physician burnout and satisfaction. Mayo Clinic proceedings, 90(4), pp. 432-440

SHANAFELT, T.D. and NOSEWORTHY, J.H., 2017. Executive Leadership and Physician Well-being: Nine Organizational Strategies to Promote Engagement and Reduce Burnout. Mayo Clinic proceedings, 92(1), pp. 129-146

STEINMANN, B., KLUG, H.J.P. and MAIER, G.W., 2018. The Path Is the Goal: How Transformational Leaders Enhance Followers' Job Attitudes and Proactive Behavior. Frontiers in psychology, 9, pp. 2338 
VAANANEN, A. et al., 2005. Are intrinsic motivational factors of work associated with functional incapacity similarly regardless of the country? Journal of epidemiology and community health, 59(10), pp. 858-863

VANDER ELST, T. et al., 2016. Perceived Control and Psychological Contract Breach as Explanations of the Relationships Between Job Insecurity, Job Strain and Coping Reactions: Towards a Theoretical Integration. Stress and health : journal of the International Society for the Investigation of Stress, 32(2), pp. 100-116

WYLIE, D.A. and GALLAGHER, H.L., 2009. Transformational leadership behaviors in allied health professions. Journal of allied health, 38(2), pp. 65-73

YUKL, G.A., 2010. Leadership in organizations. 7th ed. Upper Saddle River, N.J.: Pearson Education, Inc. 
